\title{
A Comparison of miRNAs with Proinflammatory Cytokines and Procalcitonin in the Diagnosis, Treatment, Follow Up and Prognosis of Sepsis in the Intensive Care Unit
}

Semiha Orhan ( $\nabla$ smhorhan@gmail.com )

Afyonkarahisar Sağlık Bilimleri Üniversitesi Tıp Fakültesi: Afyonkarahisar Saglik Bilimleri Universitesi

Tip Fakultesi https://orcid.org/0000-0003-2617-6197

Kemal Yetıs Gulsoy

Burdur Public Hospital

Esra Orenlili Yaylagul

Adnan Menderes University: Adnan Menderes Universitesi

Halit Bugra Koca

Afyonkarahisar University of Health Sciences: Afyonkarahisar Saglik Bilimleri Universitesi

Lutfi Yavuz

Antalya Training and Research Hospital: Antalya Egitim ve Arastirma Hastanesi

Celal Ulger

Adnan Menderes Üniversitesi: Adnan Menderes Universitesi

\section{Research Article}

Keywords: gene expression, miRNA, procalcitonin, sepsis, serum, qRT-PCR

Posted Date: August 31st, 2021

DOl: https://doi.org/10.21203/rs.3.rs-841417/v1

License: (c) (i) This work is licensed under a Creative Commons Attribution 4.0 International License.

Read Full License 


\section{Abstract}

Background: The development of sepsis, the low efficacy of antibiotics used, long-term antibiotic use, and the development of resistance to antibiotics are significant problems in patients in intensive care units. The use of biological markers is promising for the diagnosis and treatment of sepsis. In this study, proinflammatory cytokines, procalcitonin and four miRNA expressions were analyzed in a time-dependent manner in a patient group and control group, and the correlations between them were examined.

Material and Method: The study included 30 patients in the intensive care unit who were diagnosed with sepsis and applied with SOFA and APACHE-2 scoring and 30 control subjects. Serum samples were taken at 24 hours, 72 hours, and on the 7th day. Analyses according to time were made of interleukin- 1 beta, interleukin-6, interleukin-10, TNF-alpha, procalcitonin and four miRNAs (miR-122, miR-146a, miR-150, and miR-223) in the collected samples and comparisons were made between the patients and the control group.

Results: At 24 hours, a decrease was observed in the miRNA-146a, miRNA-150, and miRNA-122 values and an increase in the miRNA-223 values in the sepsis group compared to the control group. At 72 hours, a decrease was observed in the miRNA-146a, miRNA-150, miRNA-122, and miRNA-223 values in the sepsis group compared to the control group.

Conclusion: When procalcitonin and inflammatory cytokines were compared with selected miRNAs in the diagnosis, treatment follow-up, and prognosis of sepsis in the intensive care unit, a correlation between procalcitonin levels, proinflammatory cytokines and miRNA-150, miRNA-146a, and miRNA-223 was found.

\section{Introduction}

The development of sepsis is one of the most important problems in patients in the intensive care unit (ICU). The development of sepsis, the low efficacy of antibiotics used, long-term antibiotic use, and the development of resistance to antibiotics are significant problems in patients in ICUs.

The beginning of the pathophysiology of sepsis is the formation of the natural immune response to an infectious pathogen. The cellular immune response leads to the expression of cytokines, chemokines, and other inflammatory regulators. Cytokines are important in ensuring that infection remains localised and preventing systemic spread $[1,2]$.

In addition to the relationship of procalcitonin with bacterial infections, elevated levels may also be seen in conditions other than infection, such as trauma, severe burns, cardiopulmonary surgery, medullary thyroid carcinoma, autoimmune diseases, immunotherapies, post-resuscitation syndrome, acute pancreatitis, and subarachnoid bleeding. Therefore procalcitonin is an insufficient biomarker in the diagnosis of sepsis. [3,4] 
Micro RNAs (miRNAs) are recently discovered bioactive cellular molecules that have an important role in the regulation of gene expressions both in normal physiology and disease conditions [5]. Different studies related to sepsis have determined that miRNA 150, miRNA 146a, miRNA 223, and miRNA 122 expressions show significant differences [6-10]. Determination of the serum levels of these candidate miRNAs has the potential to be of guidance in diagnosis and treatment.

The aim of this study was to compare procalcitonin and proinflammatory cytokines with selected miRNAs in the diagnosis, treatment and prognosis of sepsis in ICU patients.

\section{Methods}

\section{Sample collection}

The study included patients aged $>18$ years who were diagnosed with sepsis in the Intensive Care Unit (ICU) and not taking antibiotics. Patients who were admitted to the ICU with a diagnosis of sepsis or were taking antibiotics for any infection before the diagnosis of sepsis was made in the ICU were excluded from the study even if they were diagnosed with sepsis.

From the patients in the ICU throughout the study period, a total of 30 were included for analysis, comprised of 16 females and 14 males. A control group was formed of 30 patients not diagnosed with sepsis matched to the study group. Serum samples were taken from both groups.

To evaluate the efficacy of antibiotic treatment started within 24 hours of the sepsis diagnosis, blood samples were taken at 72 hours and on the 7th day after starting antibiotics. SOFA and APACHE 2 scoring were applied to evaluate morbidity and mortality.

\section{Preparation of the serum samples}

For the miRNA assays, $10 \mathrm{cc}$ blood samples were taken into sterile tubes not containing anticoagulant (DNase-RNase free) at room temperature from both the patient and control groups. Within half an hour the serum was separated and stored at $-80^{\circ} \mathrm{C}$ until assay.

\section{RNA Extraction}

RNA extraction was performed from the serum using Trizol ${ }^{\circledR}$ LS (ThermoFisherScientific) which is used for total RNA isolation from tissue fluids. To determine the amplification efficacy of the miRNA extraction performance, $100 \mathrm{fmol}$ synthetic Caenorhabditiselegans miRNA (cel-miR-39) prepared in water not containing nuclease was added to the serum (spike-in) and the extraction procedures were applied according to the manufacturer's protocol. The RNA concentration was determined within each sample.

cDNA Synthesis and qRT-PCR

Using the TaqMan ${ }^{\circledR}$ Advanced MiRNAcDNA Synthesis kit (Thermo Fisher) for the miRNA assays, cDNA synthesis was applied from total RNA. For the assays of the cDNAs synthesised with TaqMan® Fast 
Advanced Master mix and the related miRNAs (hsa-miR-150, hsa-miR-146a, hsa-miR-223, hsa-miR-122, and cel-miR-39), the TaqMan ${ }^{\circledR}$ Advanced miRNA test systems were used. Reactions were made with the StepOne RT-PCR system (Applied Biosystem) and Relative Quantification (RQ) values were obtained. All the assays were performed 3 times.

\section{ELISA analyses of the cytokines and procalcitonin amounts}

In the measurements of serum interleukin-1 beta (IL-1 $\beta$ ), IL-6, IL-10, and TNF-a levels, the eBioscience IL$1 \beta$ High Sensitivity Human ELISA Kit was used (eBioscience, Inc. San Diego, CA, USA). The absorbance readings were made with a Chem Well 2910 ELISA reader device (Awareness Technology, Inc. Martin Hwy. Palm City, USA). For the measurement of serum procalcitonin levels, the Biovendor Human Procalcitonin ELISA Kit was used (Biovendor-Laboratornimedicina, Karasek, Brno, Czech Republic).

\section{Statistical Analysis}

Data obtained in the study were analyzed statistically using SPSS vn 23.0 software (Statistical Package for Social Sciences, IBM, Chicago, IL, USA). Conformity of the data to normal distribution was assessed using the Kolmogorov-Smirnov and Shapiro-Wilk tests. Repeated measurement variance analysis was applied to data showing normal distribution and the Friedman test to data not showing normal distribution. When a significant difference was determined as a result of the tests, multiple comparison tests were applied to determine which time measurement had created the difference.

When a difference emerged in the measurements in the repeated measurement variance analysis, Bonferroni multiple comparison tests were used for data with normal distribution. For data not showing normal distribution, when a difference emerged in the Friedman test, the Dunn test was used to determine the level of significance.

The Mann Whitney U-test was applied in comparisons between the study group and the control group, and the Wilcoxon Signed Ranks test was used in the comparisons of time-related changes. A value of $p<0.05$ was accepted as statistically significant.

\section{Results}

A total of 120 serum samples were examined as 30 samples from the control group and 90 from the study group as 30 at each of three different time points ( 24 hours, 72 hours, 7 days). Analyses were made of proinflammatory cytokines (TNF-a, IL-1 $\beta, I L-6, I L-10$ ), procalcitonin, and 4 miRNAs (miR-122, miR-146a, miR-150, miR-223). Comparisons were made between the study group and the control group, and between the time measurement points in the study group.

In the analysis of miRNA-146a expression, a significant decrease was determined in the study group compared to the control group and according to time (Figure 1A-1B). In the time related changes of the miRNA results, a statistically significant difference between the values at 24 hours and 72 hours $(p=0.005)$ and between 24 hours and 7 days $(p=0.0001)$ (Figure 1B) was determined. 
In the analysis of miRNA-150 expression, a significant decrease was determined in the study group compared to the control group at $72 \mathrm{hrs}$ and 7 days (Figure 1C). In the time related changes of the miRNA-150 results of the study group, a statistically significant difference between the values at 24 hours and 72 hours $(p=0.013)$ and between 24 hours and 7 days $(p=0.002)$ (Figure 1D) was determined.

In the analysis of miRNA-223 expression, a significant decrease was determined in the study group compared to the control group at $72 \mathrm{hrs}$ (Figure 1E). In the time related changes of the miRNA-223 results of the study group, a statistically significant difference between the values at 24 hours and 72 hours $(p=0.0001)$ and the difference between 24 hours and 7 days was not statistically significant $(p=0.166)$ (Figure 1F) was determined.

In the analysis of miRNA-122 expression, no significant difference was determined between the study group and the control group (Figure 1G). In the time-related changes of the miRNA-122 results of the study group, a statistically significant difference between the values at 24 hours and 72 hours $(p=0.041)$ and between 24 hours and 7 days $(p=0.027)($ Figure $1 H)$ was determined.

The TNF-a results of the study group were found to be statistically significantly higher than those of the control group at 72 hours and 7 days (Figure 2A). When the TNF-a results were evaluated according to time, no statistically significant difference was determined between the values at $24 \mathrm{hrs}$ and $72 \mathrm{hrs}$ $(p=0.318)$, and a statistically significant decrease was observed between 24 hours and 7 days $(p=0.049)$ (Figure 2B).

The IL-1 $\beta$ results of the study group were found to be statistically significantly higher than those of the control group at $24 \mathrm{hrs}, 72 \mathrm{hrs}$, and 7 days (Figure $2 \mathrm{C}$ ). When the IL-1 $\beta$ results were evaluated according to time, a statistically significant difference was determined between the values at $24 \mathrm{hrs}$ and $72 \mathrm{hrs}$ $(p=0.011)$, and between 24 hours and 7 days $(p=0.001)$ (Figure 2D).

The IL- 6 results of the study group were found to be statistically significantly higher than those of the control group at $24 \mathrm{hrs}, 72 \mathrm{hrs}$, and 7 days (Figure 2E). When the IL-6 results were evaluated according to time, a statistically significant difference was determined between the values at $24 \mathrm{hrs}$ and $72 \mathrm{hrs}$ $(p=0.0001)$, and between 24 hours and 7 days $(p=0.0001)$ (Figure $2 F)$.

The IL-10 results of the study group were found to be statistically significantly higher than those of the control group at $24 \mathrm{hrs}, 72 \mathrm{hrs}$, and 7 days (Figure 2G). When the IL-10 results were evaluated according to time, a statistically significant difference was determined between the values at $24 \mathrm{hrs}$ and $72 \mathrm{hrs}$ $(p=0.0001)$, and between 24 hours and 7 days $(p=0.0001)$ (Figure $2 \mathrm{H})$.

The procalcitonin results of the study group were found to be statistically significantly higher than those of the control group at $24 \mathrm{hrs}, 72 \mathrm{hrs}$, and 7 days (Figure 2l). When the procalcitonin results were evaluated according to time, a statistically significant difference was determined between the values at $24 \mathrm{hrs}$ and $72 \mathrm{hrs}(\mathrm{p}=0.026)$, and between 24 hours and 7 days $(p=0.0001)$ (Figure $2 \mathrm{~J})$. 


\section{Discussion}

Early diagnosis and evaluation of sepsis is very important for the timely treatment of this complex syndrome. Microbiological culture is the gold standard for the differentiation of sepsis from other noninfectious diseases. However, as this technique takes a long time, diagnosis and treatment are delayed. Therefore, several studies have been conducted to determine the ideal biomarker for sepsis.

The studies in the literature that have examined procalcitonin and cytokine levels to evaluate the duration of an antibiotic use in ICU patients with sepsis have not included patients using antibiotic treatment on admission to ICU [11-15]. Taking the above-mentioned literature and meta-analyses into consideration, the patients included in the current study were those being followed up in ICU who developed sepsis and who had not previously received antibiotic treatment, to be able to create a more homogenous standardised group. This study aimed to compare candidate miRNAs with procalcitonin and proinflammatory cytokines in the evaluation of the efficacy of antibiotic treatment in patients being followed up in ICU who were diagnosed with sepsis and started antibiotic treatment within the first 24 hours.

miRNAs have recently been shown to be related to the pathogenesis of inflammation and sepsis [16]. The stimulation of TLRs in the process of sepsis development plays a role in the induction of nuclear factor kappa B (NF-KB) and the expression of mitogen activated protein kinase (MAPK), monocytes, and macrophages in proinflammatory cytokines [17]. miRNAs directly target signal proteins and control NF-KB activity in immune cells, and are therefore defined as regenerators of the immune system [18]. A link between miRNA in particular and NF-KB has been shown in in-vitro studies.

It has been reported that miRNA-146a and miRNA-146b are expressed as a response to the microbial component and proinflammatory cytokines. There is a relationship of miRNA-146a and $b$ with kinase 1 genes related to II-1 receptor and 3'UTRs of TNF-related factor 6 [19]. In another study, Funahashi et al. showed that miRNA-146a has an anti-inflammatory role and reduced excessive inflammation by regulating IRAK-1 and TRAF6, thereby modulating NF-KB transcriptional activity. In an in-vitro environment, miRNA-146a has been shown to trigger severe sepsis in macrophages and to be correlated with sepsis severity at the cellular level [20].

In a study of 50 sepsis patients, 30 patients with SIRS, and a control group of 20 , Wang et al. found that the serum miRNA-146a and miRNA-223 values were significantly lower in the sepsis group compared to the SIRS group and the healthy control group, but IL-6 and CRP values were significantly high [6].

In another study that analyzed chemokines and cytokines in sepsis patients, changes in miRNA-146 were shown to be correlated with polymorphonuclear cells in peripheral blood. IL-6, which is an important inflammatory mediator in sepsis, was reported to increase, while miRNA-146 decreased [21].

The IL- 6 level increases more rapidly in sepsis and reaches a peak level at the end of the second hour. The elevated serum level lasts longer than for TNF-a and IL-1 [22]. Procalcitonin (PCT) is produced as a 
response to endotoxin or through mediators (IL-1, TNF-a, IL-6) expressed as a response to bacterial infections. It is correlated with the severity of bacterial infection $[23,24]$.

In the current study, miRNA-146a was determined to be significantly reduced in the sepsis group compared to the control group at $24 \mathrm{hrs}, 72 \mathrm{hrs}$, and 7 days of treatment, and a significant increase was determined in proinflammatory cytokine and procalcitonin levels, which was consistent with findings in the literature. In addition, a significant decrease was determined in the miRNA-146a levels in the sepsis group at $72 \mathrm{hrs}$ and 7 days compared to the values at 24 hours, and there was a significant decrease in proinflammatory cytokines and procalcitonin levels.

Schmidt WM et al showed in vivo that the miRNA-150 level decreased in peripheral blood leukocytes during acute LPS-related inflammation [25]. Roderburg $C$ et al. compared 138 patients meeting sepsis criteria and 76 healthy control subjects, and reported that the serum miRNA-150 level was slightly reduced in the sepsis group, and was therefore not suitable as a marker for the diagnosis of sepsis. However, a correlation between serum miRNA levels and hepatic and renal function disorders, and low serum miRNA-150 levels were reported to be associated with a poor prognosis [26]. Low miRNA150 levels in the circulation may cause a decrease in genes such as CXCR4 and c-Myb, which are related to both immune activation and prognosis [27]. In a study of 120 sepsis patients and 50 healthy control subjects, Ma et al. showed that miRNA-150 was significantly decreased in the sepsis group compared to the control group, and there was a negative correlation between IL-6, TNF-a, and miRNA-150 values [28].

In the current study, the miRNA-150 level was observed to be decreased in the sepsis patients compared to the control group, but at 24 hours this decrease was not statistically significant. These differences from previously published results are most probably related to the size and characteristics of the patient groups analyzed in different studies. A statistically significant decrease was seen in the miRNA-150 levels of the sepsis group after 72 hours and 7 days of sepsis treatment compared to the control group. When the sepsis antibiotic treatment was evaluated over time, a significant decrease was determined in the miRNA-150 level of the sepsis group at $72 \mathrm{hrs}$ and 7 days compared to the value at 24 hours. The proinflammatory cytokines and procalcitonin values were seen to be significantly increased at 24 hours when sepsis was diagnosed, and significantly decreased after $72 \mathrm{hrs}$ and 7 days of treatment. Roderburg et al. determined no correlation between serum miRNA-150 levels and markers of bacterial infection and inflammation such as CRP or procalcitonin [26].

In addition to miRNA-122 being known to be a miRNA associated with the liver, it has also been stated to be a biomarker in the diagnosis and prognosis of sepsis [10]. When coagulation disorder develops during the course of sepsis, the miRNA-122 level has been reported to increase.[29]. In a study of 108 sepsis patients in the first $24 \mathrm{hrs}$ of admission to ICU and 20 control subjects, miRNA-122 expression was seen to be 40 -fold higher in sepsis patients with a mortal course compared to the control group, and 6-fold higher in the patients with sepsis who survived [30].

In the current study, an increase was seen in the miRNA-122 levels at 24 hours following the diagnosis of sepsis compared to the control group, and a decrease was determined after 72 hours and 7 days of 
treatment. Within the sepsis group, there was a significant decrease in the miRNA-122 levels at 72 hours and 7 days. Moreover, a parallel decrease was seen in proinflammatory cytokines and procalcitonin levels.

In a study by Wang et al of 166 patients diagnosed with sepsis and 24 control subjects, serum samples were taken from the sepsis patients within 24 hours. There was a diagnosis of severe sepsis and septic shock in 123 patients and mild sepsis in 43. The miRNA-223 expression level was reported to be significantly higher, and the miR-499-5p, miR-122, and miR-193b levels significantly lower in the mild sepsis, severe sepsis, and septic shock patients compared to the control group. In addition, the miRNA223 expression level was statistically slightly elevated in the septic shock and severe sepsis group compared to the mild sepsis group [8].

In two separate studies of neonatal sepsis patients, Dhas et al. reported a significant decrease in plasma miRNA-223 levels compared to the control group, whereas Benz et al. found no significant difference in miRNA levels [31,32].

In the current study, an increase, but not at a significant level, was seen in the miRNA-223 levels of the sepsis group compared to the control group, a statistically significant decrease was determined after 72 hours of sepsis treatment, and on the 7th day, an increase was seen which was not statistically significant because of the increase in standard deviation.

The identification of miRNAs and their potential target genes may contribute to the understanding of the complex transcriptional programming of the natural immune system triggered by pathogens. Impairment of the mechanisms which regulate the immune system during sepsis may lead to loss of inflammation control resulting in host damage and deep immunosuppression [33].

In this study, which compared selected miRNAs with procalcitonin and inflammatory cytokines in the diagnosis, treatment follow-up, and prognosis of patients diagnosed with sepsis in ICU, a relationship was determined between miRNA-150, miRNA-146a, and miRNA-223, and proinflammatory cytokines and procalcitonin levels.

Due to the lack of reliable biomarkers of immune system dysfunction associated with sepsis, effective therapeutic interventions cannot be made for septic patients.

\section{Conclusion}

This was a preliminary study conducted in a single centre with a limited number of sepsis patients. Based on this study, by increasing the number of patients and with data obtained from comprehensive analysis systems such as all miRNA pool microarray or new-generation sequencing, new biomarkers will be able to be established for the diagnosis, treatment follow up, and prognosis of sepsis. Candidate miRNAs which have been determined in literature were investigated and some which could potentially be used as biomarkers were revealed. An increase in the number of samples and further studies of the entire miRNA 
pool will be able to both obtain more reliable data for the miRNAs studied within the scope of this study and reveal potential miRNAs to be new biomarkers. In addition, further studies evaluating the microorganisms produced in cultures taken from patients and the changes in miRNA expression values of antibiotics used in treatment will be of guidance in the evaluation of treatment success and perhaps the selection of the correct antibiotic.

\section{Declarations}

\section{Ethical Approval and Consent to participate:}

The study was conducted following the Declaration of Helsinki, and patients gave their written consent. Approval for the study was granted by the Clinical Research Ethics Committee of Suleyman Demirel University School of Medıcıne (decision no:31, dated:2016)

\section{Consent for publication}

The authors declare that this article is original, has never been published, and has not been submitted to any other journal.

\section{Availability of supporting data}

Not applicable.

\section{Consent for publication:}

Consent was obtained from all participants.

\section{Competing interests}

The authors declare no competing or financial interests.

\section{Funding}

This work was supported by generous funding from the THE SCIENTIFIC AND TECHNOLOGICAL RESEARCH COUNCIL OF TURKEY (TUBITAK) 3001 AR-GE.

\section{Authors' contributions}

The authors SO,KYG,CU,LY,EOY and HBK declared that they have no competing interests. The author SO, KYG drafted the work. SO,CU,KYG,EOY reviewed and edited the work. SO,KYG and EOY collected data and revised the figures.CU,HBK,LY substantively revised this manuscript and supervised the work. SO,KYG,LY designed and conceptualized this work. All authors read and approved the final manuscript.

\section{Acknowledgements}




\section{References}

1. Tosi MF. 2005.Innate immune responses to infection. J Allergy Clin Immunol.116(2),241-249. https://doi.org/doi: 10.1016/j.jaci.2005.05.036.

2. Werling $D$, and Jungi TW. 2003. Toll-like receptor slinking innate and adaptive immune response. Vet Immunol Immonopathol.10(91),1-12. https://doi.org/doi: 10.1016/s0165-2427(02)00228-3.

3. Tang BM, Eslick GD, Craig JC, and McLean AS.2007. Accuracy of procalcitonin for sepsis diagnosis in critically ill patients: systematic review and meta-analysis. Lancet Infect Dis.7(3)210-217. https://doi.org/doi: 10.1016/S1473-3099(07)70052-X.

4. Bréchot N, Hékimian G, Chastre J, and Luyt CE.2015. Procalcitonin to guide antibiotic therapy in the ICU. Int J Antimicrob Agent.46(1)19-24. https://doi.org/doi: 10.1016/j.jiantimicag.2015.10.012.

5. Krol J, Loedige I, and Filipowicz W.2010. The wide spread regulation of micro RNA biogenesis, function and decay. Nature Reviews Genetics.11(9)597-610. https://doi.org/doi:10.1038/nrg2843

6. Wang JF, Yu ML, Yu G, Bian JJ, Deng XM, Wan XJ, and Zhu KM.2010. Serum miR-146a and miR-223 as potential new biomarkers for sepsis. Biochem Biophys Res Commun.394(1),184-188. https://doi.org/doi: 10.1016/j.bbrc.2010.02.145

7. Vasilescu C, Rossi S, Shimizu M, Tudor S, Veronese A, Ferracin M, Nicoloso MS, Barbarotto E, Popa M, Stanciulea O, Fernandez MH, Tulbure D, Bueso-Ramos CE, Negrini M, and Calin GA. 2009.MicroRNA finger prints identify miR-150 as a plasma prognostic marker in patients with sepsis.PLoSOne. 4(10):e7405. doi: 10.1371/journal.pone.0007405.

8. Wang HJ, Zhang PJ, ChenWJ, Feng D, Jia YH, and Xie LX. 2012. Four serum microRNAs identified as diagnostic biomarkers of sepsis. J Trauma Acute Care Surg. 73: 850-854. doi: 10.1097/TA.0b013e31825a7560.

9. Benz F, Roy S, Trautwein C, Roderburg C, and Luedde T.2016. Circulating MicroRNAs as Biomarkers for Sepsis.Int. J. Mol Sci. 17,(78),1-17 doi: 10.3390/ijms17010078.

10. Wang HJ, Yu B, Deng J, Jin Y, and Xie L.2014. Serum miR-122 correlates with short-term mortality in sepsis patients. Crit Care. 704,(6),12-18. doi: 10.1186/s13054-014-0704-9

11. Hochreiter M, Köhler T, Schweiger AM, Keck FS, Bein B, von Spiegel T, Schroeder S. Procalcitonin to guide duration of antibiotic therapy in intensive care patients: a randomized prospective controlled trial. Crit Care.2009;13(3):R83. doi: 10.1186/cc7903.

12. Schroeder S, Hochreiter M, Koehler T,Schweiger AM, Bein B, Keck FS, and Von Spiegel T .2009.

Procalcitonin (PCT)-guided algorithm reduces length of antibiotic treatment in surgical intensive care patients with severe sepsis: results of a prospective randomized study. Langenbecks Arch Surg. 394(2):221-226. doi: 10.1007/s00423-008-0432-1.

13. Branche AR, Walsh EE, Vargas R, Hulbert B, Formica MA, Baran A, Peterson DR, and Falsey AR .2015. Serum Procalcitonin Measurement and Viral Testingto Guide Antibiotic Use for Respiratory Infections 
in Hospitalized Adults: A Randomized Controlled Trial. J Infect Dis. 212(11):1692-700.doi: 10.1093/infdis/jiv252.

14. Kopterides P, Siempos II, Tsangaris I, Tsantes A, and Armaganidis A.2010. Procalcitonin-guided algorithms of antibiotictherapy in theintensive care unit: a systematic review and meta-analysis of randomized controlled trials. Crit Care Med.38(11):2229-2241. doi:

10.1097/CCM.0b013e3181f17bf9.

15. Rhee C. 2016. Using Procalciton into Guide Antibiotic Therapy. Open Forum Infect Dis. 7;4(1)ofw249 doi: 10.1093/ofid/ofw249.

16. Sonkoly E, Stahle M, and Pivarcsi A. 2008. Micro RNAs and immunity : novel players in the regulation of normal immune function and inflammation. Sem in Cancer Biol.18: 131-140. doi:

10.1016/j.semcancer.2008.01.005.

17. Tsujimoto H, Ono S, Efron PA, Scumpia PO, Moldawer LL, and Mochizuki H. 2008. Role of Toll-like receptors in the development of sepsis. Shock. 29(3):315-321. doi:

10.1097/SHK.0b013e318157ee55.

18. Ma X, Becker BLE, Barker JR, and Li Y. 2011. MicroRNAs in NF-kappa-B signaling. J Mol Cell Biol.3(3):159-166.doi: 10.1093/jmcb/mjr007.

19. Taganov KD, Boldin MP, Chang KJ, and Baltimore D. 2006. NF-kappaB-dependent induction of microRNA miR-146, an inhibitor targeted to signaling proteins of innate immune responses. Proc Natl Acad Sci USA. 103: 12481-12486. doi: 10.1073/pnas.0605298103.

20. Funahashi Y, Kato N, Masuda T, Nishio F, Kitai H, Ishimoto T, Kosugi T, Tsuboi N, Matsuda N, Maruyama S, and Kadomatsu K . 2019. miR-146a targeted to splenic macrophages prevents sepsisinduced multiple organ injury. Laboratory Investigation.99:1130-1142.doi: 10.1038/s41374-0190190-4.

21. Zhou J, Chaudhry H, Zhong Y, Ali MM, Perkins LA, Owens WB, Morales JE, McGuire FR, Zumbrun EE, Zhang J, Nagarkatti PS, and Nagarkatti M. 2015. Dysregulation in microRNA expression in peripheral blood mono nuclear cells of sepsis patients is associated with immunopathology. Cytokine.71(1):89100.doi: 10.1016/j.cyto.2014.09.003

22. Song M, and Kellum JA.2005. Interleukin-6, CritCare Med,33,(12),463-5.doi: 10.1097/01.ccm.0000186784.62662.a1.

23. Gogos CA, Drosou E, Bassaris HP, and Skoutelis A. 2000. Pro- versus anti-inflammatory cytokine profile in patients with severe sepsis: a marker for prognosis and future therapeutic options, J. Infect. Dis.181,(1),176-180.doi: 10.1086/315214.

24. Brodská H, Malíčková K, Adámková V, Benáková H, Št́astná MM, and Zima T.2013. Significantly higher procalcitonin levels could differentiate Gram-negative sepsis from Gram-positive and fungal sepsis. Clin Exp Med.13:165-170. doi: 10.1007/s10238-012-0191-8.

25. Schmidt WM, Spiel AO, Jilma B, Wolzt M, and Müller M. 2009. Invivo profile of the human leukocyte microRNA response to endotoxemia. Biochem Biophys Res Commun.380,(3),437-441.doi: 10.1016/j.bbrc.2008.12.190. 
26. Roderburg C, Luedde M, Cardenas DV, Vucur M, Scholten D, Frey N, Koch A, Trautwein C, Tacke F, and Luedde T.2013. Circulating MicroRNA-150 Serum LevelsPredictSurvival in Patientswith Critical Illness and Sepsis. PLoS ONE. 8(1): e54612. doi: 10.1371/journal.pone.0054612.

27. Xiao C, Calado DP, Galler G, Thai TH, and Patterson HC. 2007. MiR-150 controls B cell differentiation by targeting he transcription factor c-Myb. Cell. 131: 146-159. doi: 10.1016/j.cell.2007.07.021.

28. Ma Y, Liu Y, Hou H, Yao Y, and Meng H. 2018. MiR-150 predicts survival in patients with sepsis and inhibits LPS-induced inflammatory factors and apoptosis by targeting NF-kB1 in human umbilical vein endothelial cells. Biochem Biophys Res Commun. 500(3):828-837. doi: 10.1016/j.bbrc.2018.04.168.

29. Wang HJ, Deng J, Wang J.Y, Zhang PJ, Xin Z, Xiao K, Feng D, Jia YH, Liu YL, and Xie LX. 2014. Serum miR-122 levels are related to coagulation disorders in sepsis patients. Clin. Chem. Lab. Med. 52, 927-933. doi: 10.1515/cclm-2013-0899.

30. Rahmel T, Schäfer ST, Frey U.H, Adamzik M, and Peters J.2018. Increased circulating microRNA-122 is a biomarker for discrimination and risk stratification in patients definedby sepsis-3 criteria. PLOS ONE. 13(5):e0197637.doi: 10.1371/journal.pone.0197637.

31. Dhas BB, Dirisala VR, and Bhat BV.2018. ExpressionLevels of CandidateCirculatingmicroRNAs in Early-Onset Neonatal Sepsis Compared With Healthy Newborns. Genomics Insights.11:1178631018797079. doi: 10.1177/1178631018797079.

32. Benz F, Tacke F, Luedde M, Trautwein C, Luedde T, Koch A,and Roderburg C. 2015. Circulating microRNA-223 serum levels do not predict sepsis or survival in patients with critical illness. Dis Markers. 384208. doi: 10.1155/2015/384208.

33. Hotchkiss RS, and Nicholson DW.2006. Apoptosis and caspases regulate death and inflammation in sepsis. Nat Rev Immunol. 6: 813-822. doi: 10.1038/nri1943.

\section{Figures}


(A)

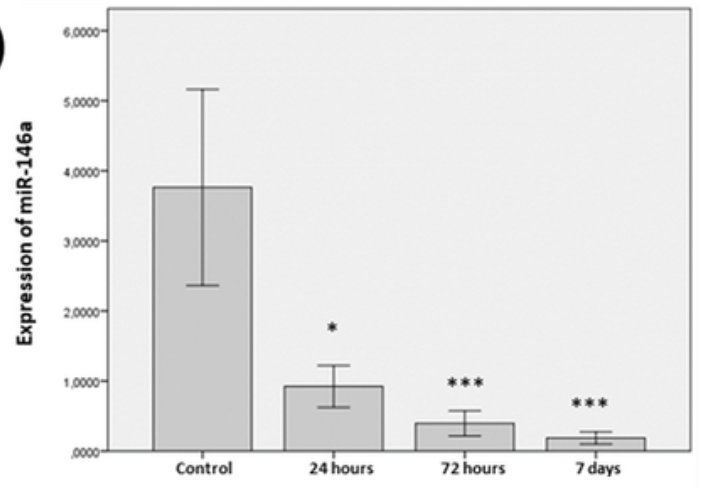

(c)

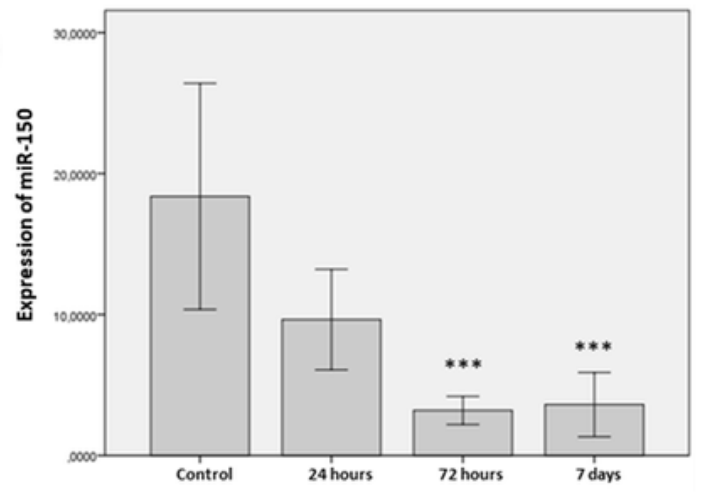

(E)

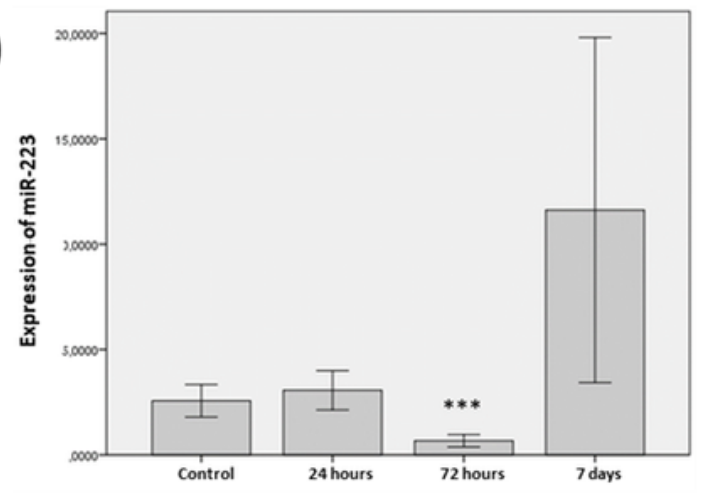

(G)

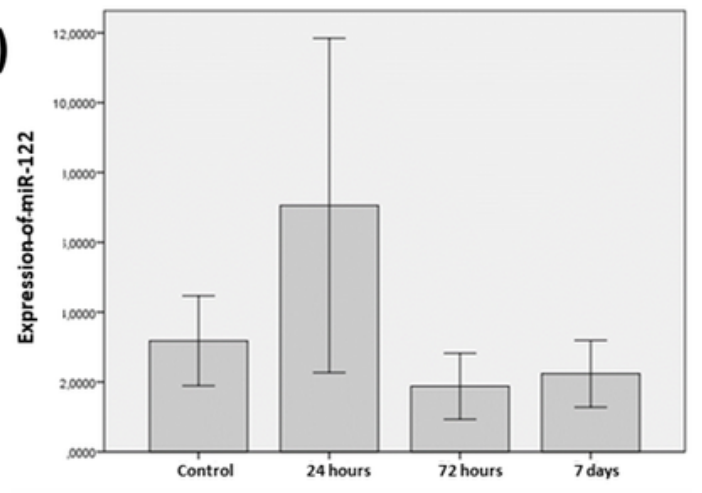

(B)

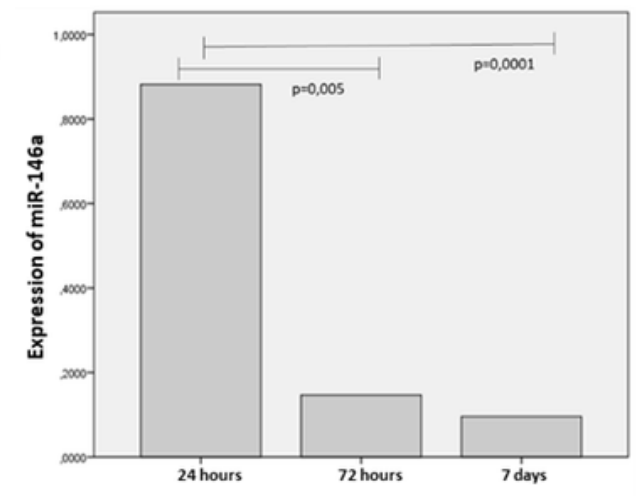

(D)

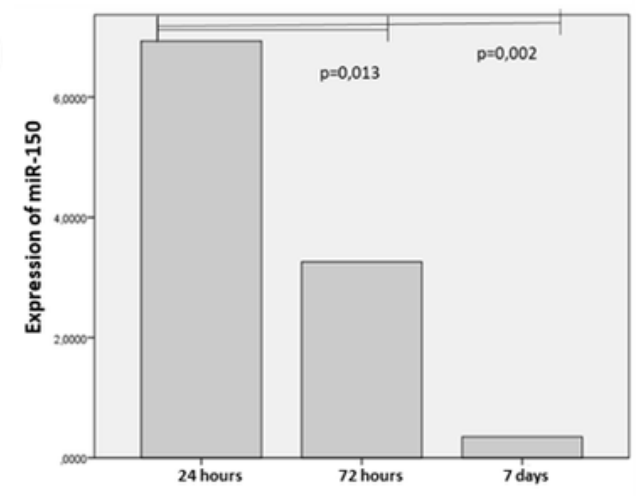

(F)

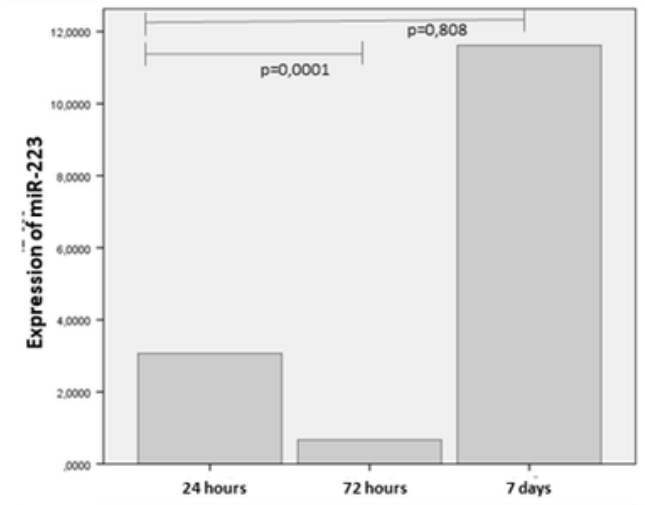

(H)

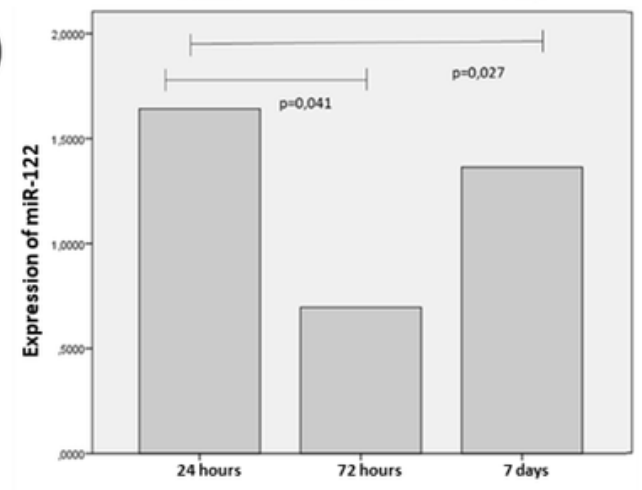

\section{Figure 1}

Images on the left column show the relative expressions of miRNAs compared with the healthy control groups and the right column show that time-dependent miRNA expression change. (A) miR-146a significantly reduced in 24th $\mathrm{h}$, 72th $\mathrm{h}$ and 7th day (B)miR-146a significantly reduced after treatment (C) miR-155 significantly reduced in 72th $\mathrm{h}$ and 7th day. (D) miR-155 signicantly reduced after treatment (E) 
miR-223 significantly reduced in 72th $\mathrm{h}(\mathrm{F})$ miR-223 significantly reduced in 72th $\mathrm{h}(\mathrm{G})$ miR-122 expressions were not changed ${ }^{*} p<0.05,{ }^{* *} p<0.01,{ }^{* * *} p<0.001$

(A)

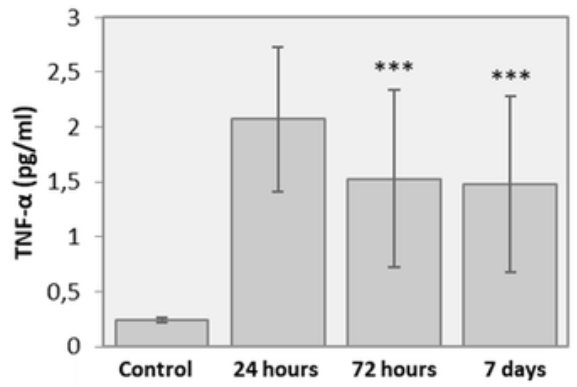

(C)

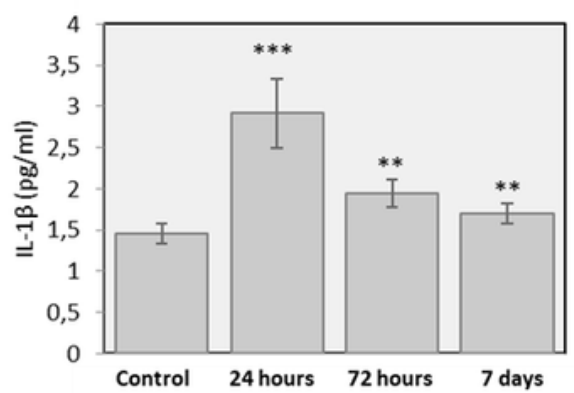

(E)

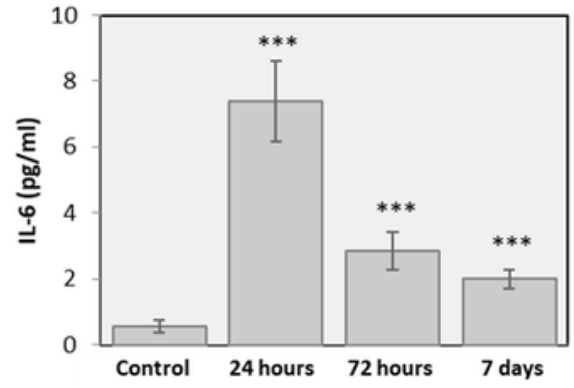

(G)

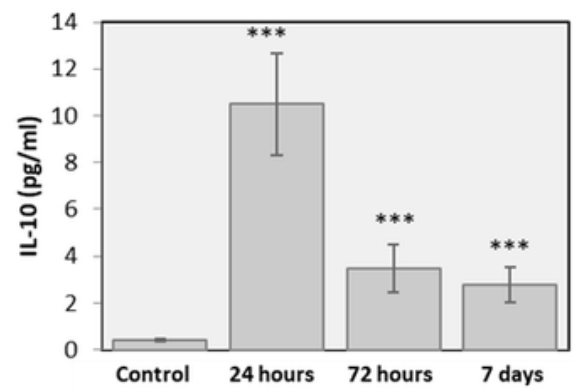

(I)

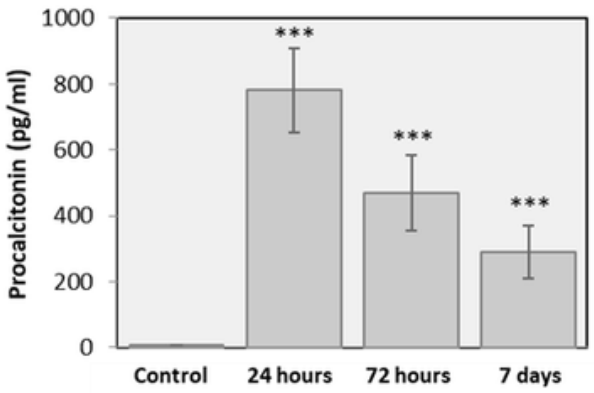

(B)

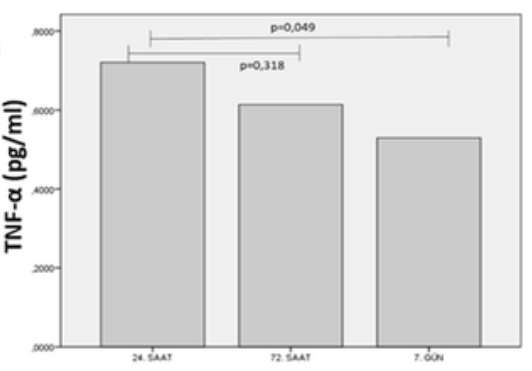

(D)

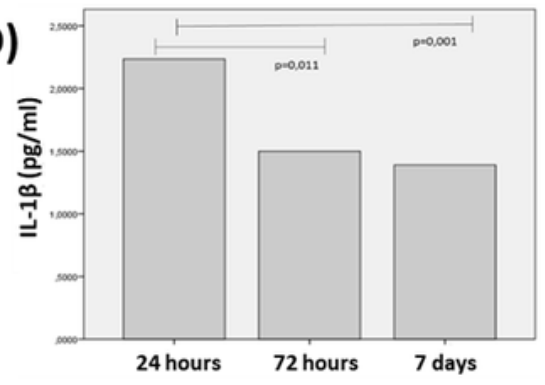

(F)

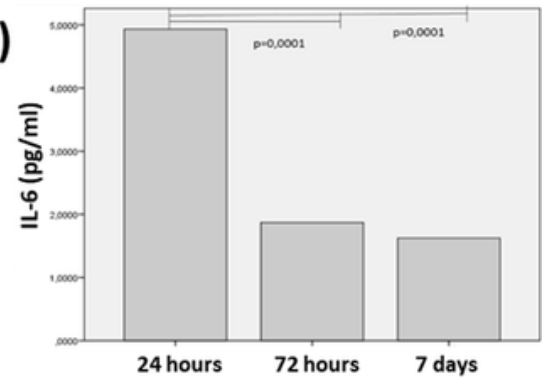

(H)

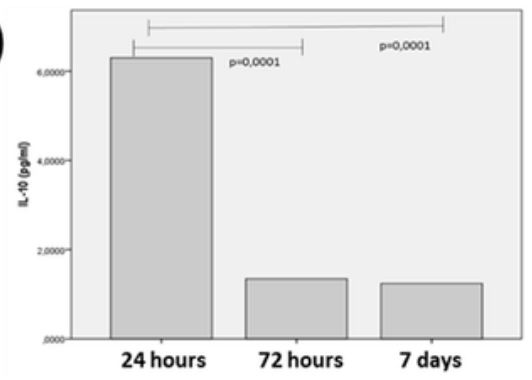

(J)

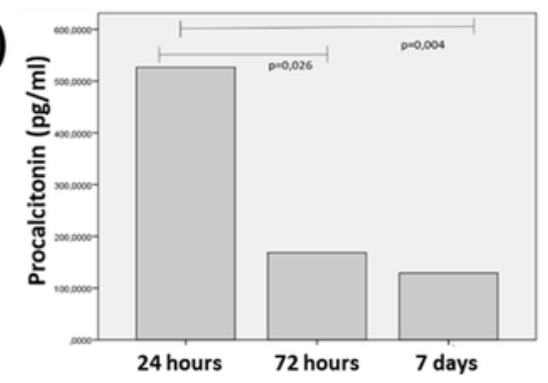

\section{Figure 2}

Images on the left column show the levels of inflammatory cytokines and procalcitonin compared with the healthy control groups and the right column show that time-dependent inflammatory cytokines and procalcitonin levels change. (A-C-E-G-I) inflammatory cytokines and procalcitonin levels were significantly 
higher than the control group (B) TNF-a level significantly reduced in 7th day (D-F-H-J) IL-1 $\beta$, IL-6, IL-10 and procalcitonin levels significantly reduced in 72 nd hour and 7 th day. ${ }^{*} p<0.05,{ }^{* \star} p<0.01,{ }^{\star \star \star} p<0.001$ 